e-Neuroforum 2013 · 4:31-38

DOI 10.1007/s13295-013-0042-4

๑) Springer-Verlag 2013
F. Helmchen ${ }^{1} \cdot$ M. Hübener ${ }^{2}$

${ }^{1}$ Brain Research Institute, University of Zurich, Zurich

${ }^{2}$ Max Planck Institute of Neurobiology, Martinsried

\title{
Neuronal networks in the spotlight
}

\section{Deciphering cellular activity patterns with fluorescent proteins}

\section{Introduction}

A fundamental goal of neuroscience is to elucidate the principles of signal processing in neuronal networks. In this context, a distinction is often made between the activity in local microcircuits-the complex interconnected networks of neighboring excitatory and inhibitory neurons, for instance, in a cortex region or a particular brain nucleus-and the activity in large-scale networks of communicating brain regions. Local microcircuits integrate input signals in a complex manner and then transfer information further to downstream networks, depending on the evoked activity patterns in projecting neurons. A central idea is that specific computational operations are carried out while signals flow through local networks, and that uncovering the underlying cellular and molecular mechanisms will be crucial for understanding information processing in the brain. However, the complexity of neuronal networks-with regard to both anatomical circuit wiring and the possibilities of functional modulation - cannot be underestimated. This notion is, for example, illustrated by considering the very different dynamic states of cortical networks under anesthetized and awake conditions.

The first, supposedly easy, step is the phenomenological description of neuronal network activity under relevant conditions, i.e., in situations in which the brain is performing a task, when it is "in action." However, the experimental possibilities for such a descriptive approach have been scarce in the past, and the development of suitable tools is still a major challenge. On the macroscopic level, the activity of particular regions of the human brain can be measured in various situations by functional magnet resonance imaging (fMRI). By contrast, the methods for measuring dynamic activity patterns in local neuronal networks with cellular resolution have been limited. Although the basic properties of individual cell types and molecular events underlying the communication between neurons have been intensively studied over the past decades, the tremendous complexity of neuronal networks with still largely unknown wiring schemes and modulation principles has precluded direct insights into their dynamic properties. With electrophysiological recordings it is possible to sample the activity of a few, but not all, neurons within a local network. Moreover, to date it was hardly possible to measure the activity of the same neurons repeatedly over a long time period to examine functional changes of network dynamics, for example, during learning. Such direct measurement of activity patterns in local neuronal circuits, in particular under in vivo conditions and over long time periods, would also be of high importance for improving our understanding of malfunctions of neuronal networks during brain diseases. Here, we review recent progress in optical imaging of neuronal population activity that has opened a multitude of new pos- sibilities to study and explain the relationship between neuronal network activity and fundamental brain functions such as sensory perception, behavior control, and decision making.

\section{Genetically encoded calcium indicators}

For several decades, neuroscientists have utilized the combination of activity-dependent fluorescent indicators and highresolution microscopy techniques to visualize dynamic excitation patterns of neuronal cell populations. Until the end of the last century, most indicators were small organic molecules that change their fluorescence properties in response to membrane potential changes or binding to calcium ions, for example, following calcium influx through voltage-sensitive calcium channels during an action potential. Following the development of two-photon microscopy around 1990, these sophisticated small molecule indicators enabled high-resolution imaging of neurons, even in vivo, in intact animal brains. However, staining procedures remained challenging and repeated measurements over several days or longer time periods were nearly impossible.

Shortly after the cloning and widespread distribution of the green fluorescent protein (GFP), several groups started developing activity indicators based on GFP variants. The first genetically encoded calcium indicator "Cameleon" was re- 


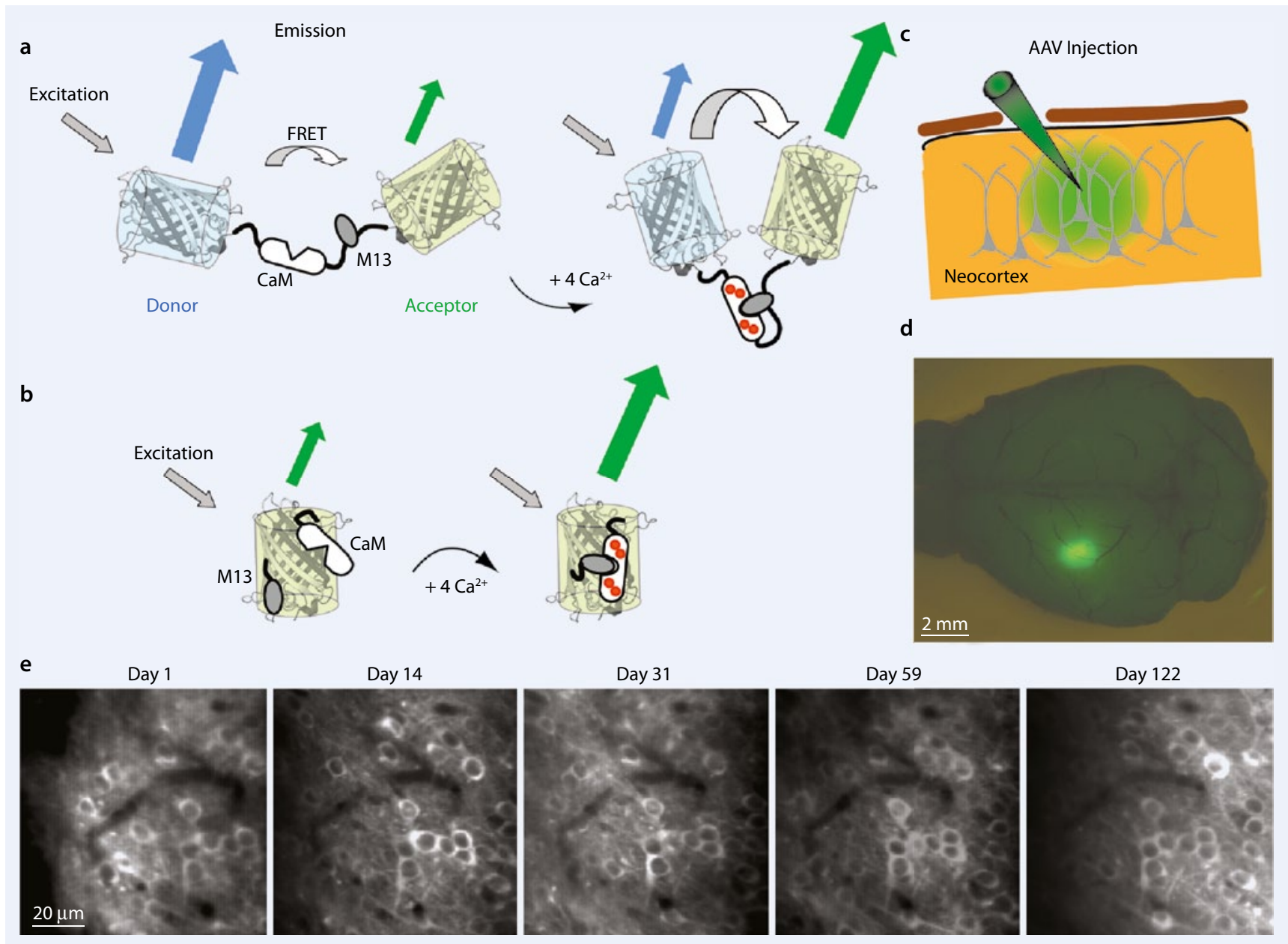

Fig. $1 \Delta$ Functionality of genetically encoded calcium indicators and stable expression in cortical neurons. a One important class of indicators is based on the FRET effect between two coupled fluorescent proteins. Energy transfer between the donor and the acceptor protein depends on their relative distance and orientation. Binding of calcium ions to the linker-protein calmodulin (CaM) causes an interaction with the CaM-binding protein domain M13 and induces a conformational change that brings the fluorescent proteins closer together. As a consequence, FRET increases, resulting in a measureable change in relative fluorescence emission strengths. Besides the example of CaM-based indicators shown here, other FRET indicators exist in which the calcium-binding linker domain is based on the muscle protein troponin C. $\mathbf{b}$ Another indicator class consists of single fluorescent proteins with a built-in calcium sensor, so that calcium binding induces a fluorescence intensity change. c Virus-mediated expression of genetically encoded calcium indicators. Virus-containing solution (some tens to hundreds of nanoliters) is injected into the mouse neocortex using a thin glass pipette. $\mathbf{d}$ Example of large-scale expression of the calcium indicator YC3.60 following injection of an adeno-associated virus (AAV) vector. e Two-photon images of YC3.60-expressing neurons in the cortical layer $2 / 3$ taken at different time points. The same neurons could be observed in repeated experiments in anesthetized animals over several months. (d,e Modified from [6, 8])

ported in 1997 [10]. It consists of two fluorescent proteins (FPs), linked via a calcium-binding protein domain (calmodulin). Binding of calcium to calmodulin triggers a conformational rearrangement that alters the distance and orientation of the FPs, which in turn leads to an optically detectable change of the fluorescence resonance energy transfer (FRET) between the FPs (• Fig. 1). Over the years, FRETbased calcium indicators have been varied and improved and they form an important class of protein sensors today. Anoth- er class consists of single fluorescent proteins with a built-in calcium-binding domain, such that their fluorescence intensity is modulated depending on the intracellular calcium concentration. The most important members of this group are the so-called GCaMP proteins [12], a large palette of which is now available. The most important specifications of genetically encoded calcium indicators are their calcium-binding affinity (with typical dissociation constants in the nanomolar range), the dynamic range of attain- able fluorescence changes, and their kinetic properties that influence the time course of fluorescence change following calcium influx. For more details about the most important genetically encoded calcium indicators, we refer readers to recent reviews $[4,5]$.

Unlike classic small-molecule calcium indicators, genetically encoded indicators can be expressed in neurons by various means. The respective genes can be introduced into the organism either by direct gene transfer (e.g., by in utero electro- 
poration in mice), by creating transgenic animals, or by using viral vectors. For viral delivery, adeno-associated viruses (AAV) have found widespread application (• Fig. 1). Appropriate promoters permit neuron-specific expression of indicator genes, and selected neuronal subtypes can be targeted using conditional expression methods. Protein concentrations typically increase during the first weeks after virus injection, ideally reaching an expression level that remains stable over several months. Since cell nuclei are usually devoid of indicator protein, cells appear as fluorescent circles in the microscope images (• Fig. 1). Increasing nuclear fluorescence levels are not a good sign, as they indicate the beginning of the deterioration of cell physiology and possibly toxicity, thus shortening the maximal time window for the experiment.

A critical parameter of genetically encoded calcium indicators is their sensitivity to action-potential-evoked calcium transients. Ideally, they should be sensitive enough to report single action potentials in vivo and display little saturation during bursts of action potentials. The sensitivity can be tested in calibration experiments by simultaneous electrical recordings from individual test neurons. Meanwhile, the newest genetically encoded calcium indicators have reached a sensitivity that is comparable to the best synthetic indicators. Thus, it can be expected that they will be heavily used in the near future.

\section{In vivo imaging with calcium indicators}

Genetically encoded calcium indicators can be applied in various ways and on different spatial scales. On the macroscopic level, large-scale signal spread across several cortical areas-for instance, over an entire hemisphere-can be visualized using fast camera systems. Similar to classic experiments with voltage-sensitive dyes, large-scale cortical activity patterns and functional maps may be analyzed in this way. Stable expression of indicator proteins allows for repeated measurements from the same animal [9], which should provide new insights into the reorganization of cortical dynamics, for instance, during learning. Similarly, chronic mon- itoring of individual neurons in local microcircuits over long-term periods (weeks to month) is now possible with two-photon microscopy. To this end, a small piece of the skull is surgically removed and replaced by a permanent glass window. The virus injection can also be made during this procedure. Additionally, a small head-post is usually secured to the skull, to enable fixation of the animal's head under the two-photon microscope. Typically, water-immersion objectives with $16 \times$ to $40 \times$ magnification and a high numerical aperture are used, allowing simultaneous imaging of tens to hundreds of cells within 100-500- $\mu \mathrm{m}$ field of views. The vasculature system provides ideal landmarks for re-identification of the same cells during chronic experiments in the same animal.

Over the past decade, long-term in vivo imaging of morphological changes, for example, of synaptic structures, has been carried out frequently. Genetically encoded calcium indicators now additionally enable long-term tracking of the functional properties of neurons. Of special interest is the study of stability and plasticity of responses to sensory stimuli (such as visual or tactile stimuli) as well as the correlation of cellular activity with movement patterns or behavior. In the following, we provide examples of initial studies of this type, which addressed the question of the stability of neuronal activity in particular.

\section{Stability and plasticity of neuronal activity}

The first study in which the same neurons were imaged repeatedly using a genetically encoded calcium indicator was carried out by Mank et al. [7]. These authors used the calcium indicator TNXXL, which, as in Cameleons, is based on FRET changes, but instead of calmodulin as calcium-binding domain contains a variant of troponin $\mathrm{C}$, the calcium sensor in muscle fibers. TN-XXL was stably expressed in mouse visual cortex neurons following in utero electroporation, and cellular responses to visual stimuli were measured repeatedly in the anesthetized animal. Orientation turning of the same neurons was found to remain stable over the entire experiment period (2-3 weeks)
e-Neuroforum 2013 · 4:31-38

DOI 10.1007/s13295-013-0042-4

C) Springer-Verlag 2013

\section{F. Helmchen · M. Hübener \\ Neuronal networks in the spotlight. Deciphering cellular activity patterns with fluorescent proteins}

\section{Abstract}

The brain's astounding achievements regarding movement control and sensory processing are based on complex spatiotemporal activity patterns in the relevant neuronal networks. Our understanding of neuronal network activity is, however, still poor, not least because of the experimental difficulties in directly observing neural circuits at work in the living brain (in vivo). Over the last decade, new opportunities have emerged-especially utilizing two-photon microscopy - to investigate neuronal networks in action. Central to this progress was the development of fluorescent proteins that change their emission depending on cell activity, enabling the visualization of dynamic activity patterns in local neuronal populations. Currently, genetically encoded calcium indicators, proteins that indicate neuronal activity based on action potential-evoked calcium influx, are being increasingly used. Long-term expression of these indicators allows repeated monitoring of the same neurons over weeks and months, such that the stability and plasticity of their functional properties can be characterized. Furthermore, permanent indicator expression facilitates the correlation of cellular activity patterns and behavior in awake animals. Using examples from recent studies of information processing in the mouse neocortex, we review in this article these fascinating new possibilities and discuss the great potential of the fluorescent proteins to elucidate the mysteries of neural circuits.

\section{Keywords}

Neural network $\cdot$ Neocortex $\cdot$ Calcium indicator · Two-photon microscopy . Fluorescent protein

- Fig. 2). While this result was not unexpected, it nonetheless directly showed for the first time that fundamental properties of receptive fields of cortical neurons remain stable over longer time periods. Moreover, these proof-of-principle experiments demonstrated that the use of genetically encoded calcium indicators allows the functional properties of individual neurons to be tracked over several weeks, in contrast to small-molecule indi- 

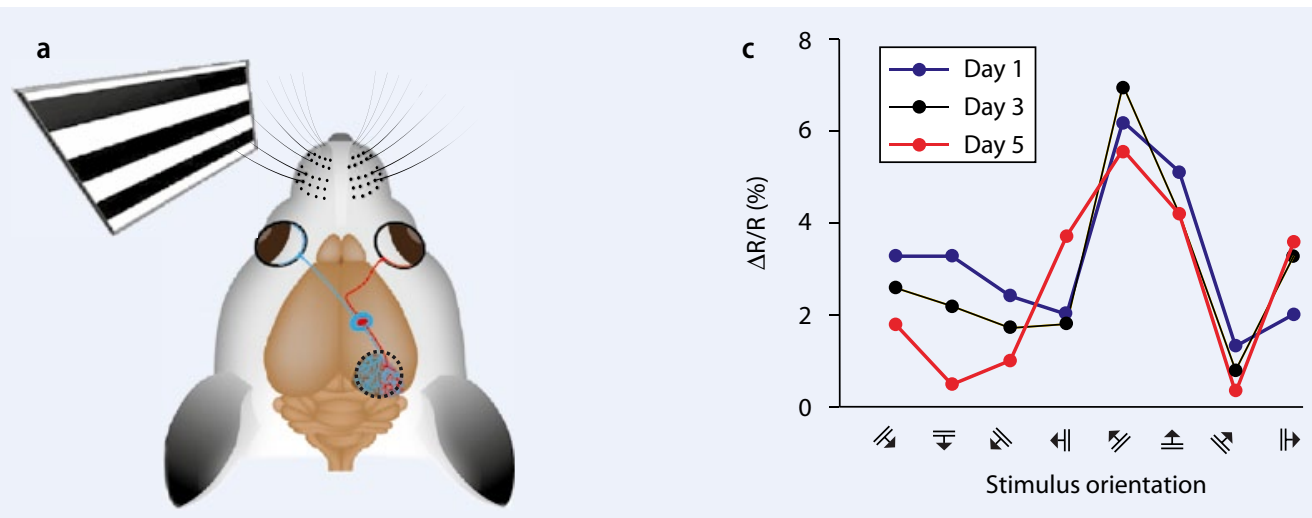

b Day 1 Day 3 Day 5
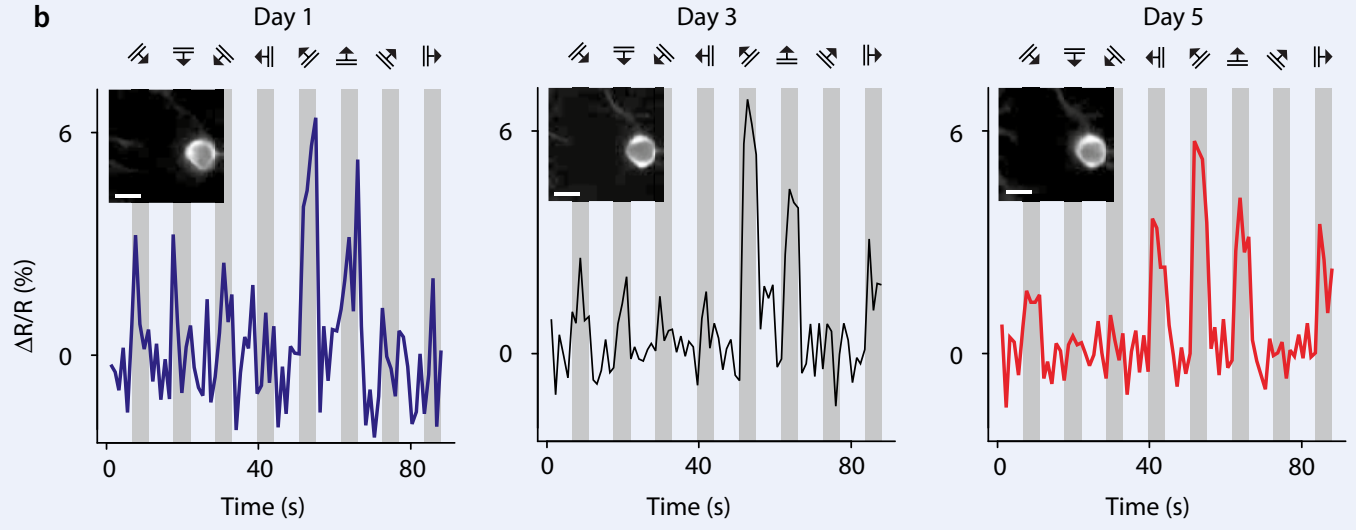

Fig. $2 \Delta$ Repeated imaging of stimulus-evoked responses of a neuron in the mouse visual cortex using a genetically encoded calcium indicator (TN-XXL; adapted from [7]). a Schematic of experimental approach. After implantation of a glass window (dotted circle) above the primary visual cortex (V1) of the right hemisphere, calcium transients in layer 2/3 neurons were measured during anesthesia using two-photon microscopy. Drifting gratings of various orientations were presented as stimuli in the contralateral visual field. $\mathbf{b}$ Responses of an example neuron (small image; scale bar $10 \mu \mathrm{m}$ ) to various orientations and directions of the visual stimulus at three time points. The relative change in donor- and acceptor-fluorescence of the FRETbased indicator $(\Delta R / R)$ is shown. Drifting grating stimuli were presented during the periods indicated by gray bars. Traces are averages of six stimulus presentations. c The orientation tuning curve of the neuron remained overall stable over the duration of the experiment

cators with which repeated measurements have been possible for a few days at most.

In another study, neuronal responses in the primary somatosensory cortex (S1) were measured upon mechanical whisker stimulation [8]. In these experiments, the FRET-indicator Yellow Cameleon 3.60 (YC3.60) was used. Through an implanted cranial window, sensoryevoked activity of S1 neurons in cortical layer 2/3 (L2/3) was repeatedly measured under anesthesia, first during a baseline period with all whiskers intact. Interestingly, activity levels in the neuronal population were quite heterogeneous, with many low-, some mid-, and a few highly responsive cells (• Fig. 3 ). This heterogeneous distribution remained stable from one day to another, suggesting that differences in neuronal excitability and synaptic activation remain stable over time. After several repeated baseline measurements, all whiskers except for one were trimmed (and re-trimmed over a few weeks), causing changes in the sensory inputs from trimmed whiskers and the non-trimmed (spared) whisker. Whisker-trimming is a classic so-called deprivation protocol that reduces sensory input and thereby induces plastic changes in the brain, especially in the affected neocortical regions. The responses of S1 neurons to stimulation of the spared whisker versus the neighboring trimmed whiskers were monitored over several weeks, establishing the first experiments in which individual neurons could be followed over a long time after the induction of plasticity. The results showed that responses evoked by stimulation of the trimmed whisker were generally reduced, whereas stimulation of the spared whisker evoked stronger responses on av- erage. However, changes in responsiveness for the spared whisker were not homogeneous across cell classes. While initially low-responsive cells became more active, the activity of initially high-responders tended to decrease (• Fig. 3). Further analysis of such redistribution of neuronal population activity-e.g., following changes in sensory inputs or in mouse models of brain diseases like Alzheimer's disease or stroke-may help to reveal principles of functional reorganization of neuronal networks during adaptation and regeneration.

\section{Network activity and behavior}

Activity patterns in neuronal networks eventually need to be interpreted within a relevant behavioral context. For experiments in awake, behaving animals, genet- 

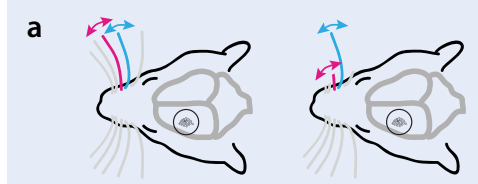

b
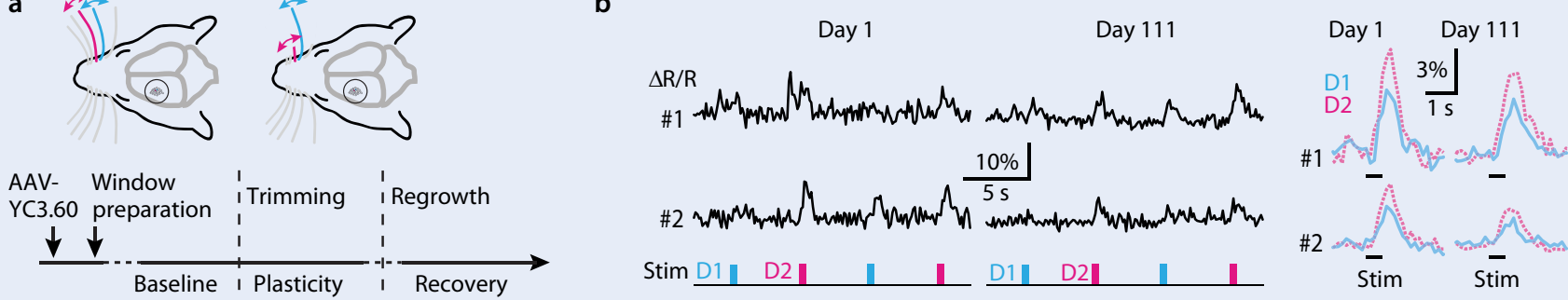

C

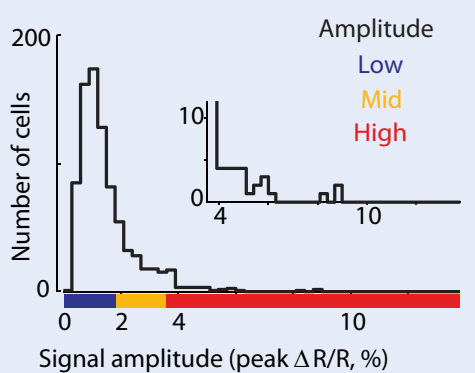

d

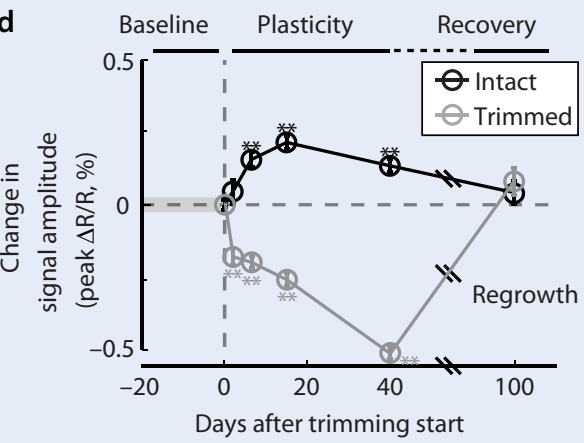

e

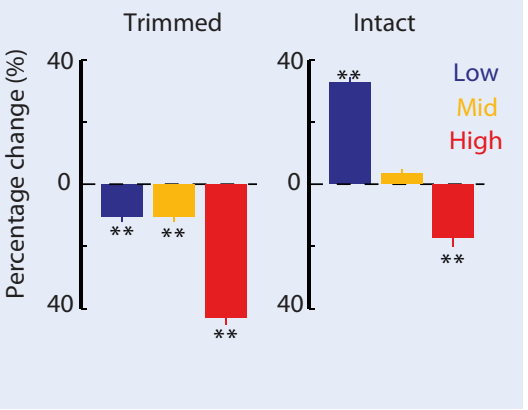

Fig. $3 \Delta$ Reorganization of neuronal activity following sensory deprivation. a Experimental protocol for studying plasticity of neuronal network activity induced by unilateral trimming of all but one whisker in the primary somatosensory cortex of the mouse using YC3.60. $\mathbf{b}$ Example of calcium transients of two cortical neurons, evoked by mechanical deflection of the D1 and D2 whisker, respectively (left, raw signals; right, averaged traces). The same cells were measured before, during, and after whisker trimming. $\mathbf{c}$ Distribution of signal amplitudes in response to whisker stimulation. Based on this distribution, neurons were classified according to their response strengths in low-, mid-, and high-responders. $\mathbf{d}$ Changes in signal amplitude (mean of all neurons) for the spared and the neighboring trimmed whisker over the entire time course of the experiment. e Percentage change in signal amplitude for spared and trimmed whisker, divided into the three functional classes. Note the redistribution of activity among the different classes. (Adapted from [8])

ically encoded calcium indicators again provide advantages compared to synthetic dyes. Although activity measurements in awake animals are possible using smallmolecule indicators, a major disadvantage is that only a single or very few calcium imaging experiments can be performed following the time-consuming habituation of an animal to the experimental setup and the behavioral task training.

Independent of the method chosen for labeling neurons with a calcium indicator, optical recordings in awake animals pose particular experimental challenges. In the last few years, several methods have been developed, principally employing two different approaches: Either the animal is more or less free to move, and optical fibers or miniaturized microscopes are attached to the skull for measurements, or the animal is head-restrained with a headpost after appropriate habituation so that imaging can be performed with a standard two-photon microscope. Dombeck and colleagues recently developed an elegant method to carry out imaging experiments on head-fixed, awake animals, following a principle that had already been successfully used to analyze motion processing in insects: The head-fixed animal (typically a mouse) rests on a treadmill, constructed from an air-supported, free-floating Styrofoam ball [1]. The inertia and friction of the ball are chosen such that the animal can easily run in two dimensions on the surface of the ball (• Fig. 4). The rotation speed and direction are captured by optical sensors and can be used, for instance, to control the movement patterns of a virtual visual environment.

This experimental setting is especially suitable for studying network activity during navigation in a visual environment (which is of particular interest in the hippocampus). Moreover, this method can reveal interactions between motor activity and sensory inputs. A key advantage of such experiments is that neuronal activity patterns can be compared under "open-loop" and "closed-loop" conditions, as has been recently applied by Keller and colleagues to study how mo- tor activity affects responses of neurons in the mouse primary visual cortex using the genetically encoded calcium indicator GCaMP3 [3]. Previous electrophysiological recordings had already revealed that running has a modulatory effect and causes elevated firing rates in many neurons, without changes in stimulus selectivity, that is, orientation preference [11]. Keller and colleagues could furthermore show that running alone already leads to a strong activation of neurons in the primary visual cortex, which was noticeable even in complete darkness. Surprisingly, these data demonstrated that in many neurons motor activity has a higher impact on the response magnitude than passive perception of moving visual stimuli during rest (• Fig. 4). A possible functional role of such strong movement-related signals could be elucidated in additional experiments: Brief interruptions of the feedback between the ball rotation and the visual virtual environment, for example, momentarily stopping the optical flow, caused strong responses in many 


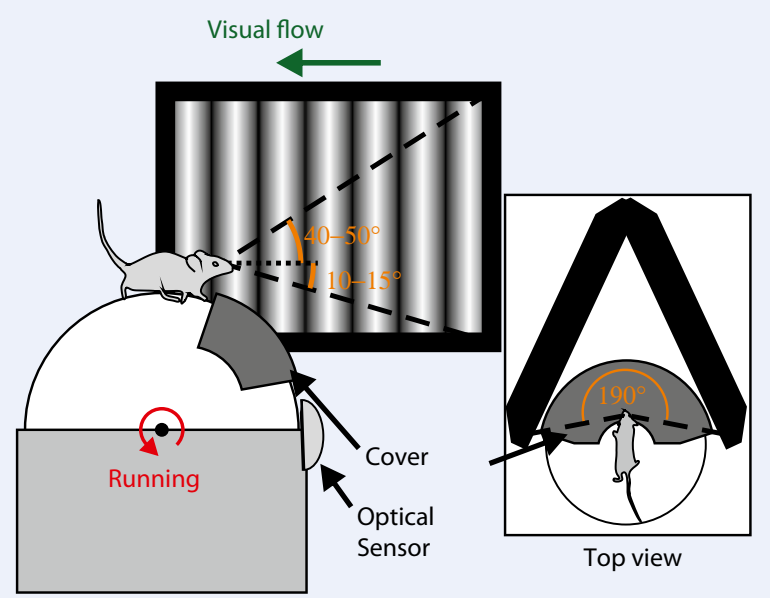

b

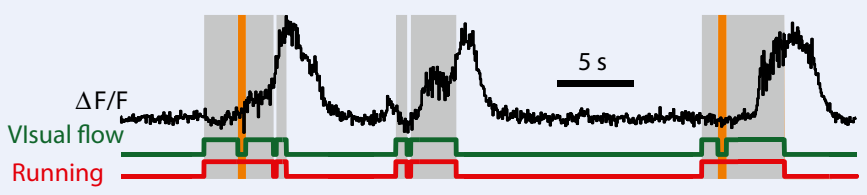

Passive stimulation

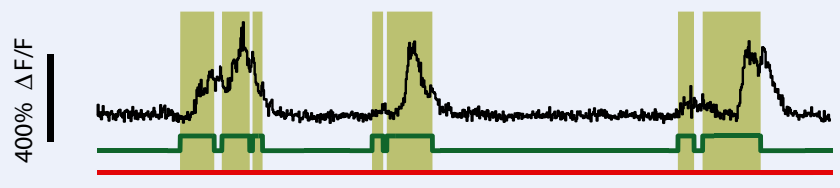

c

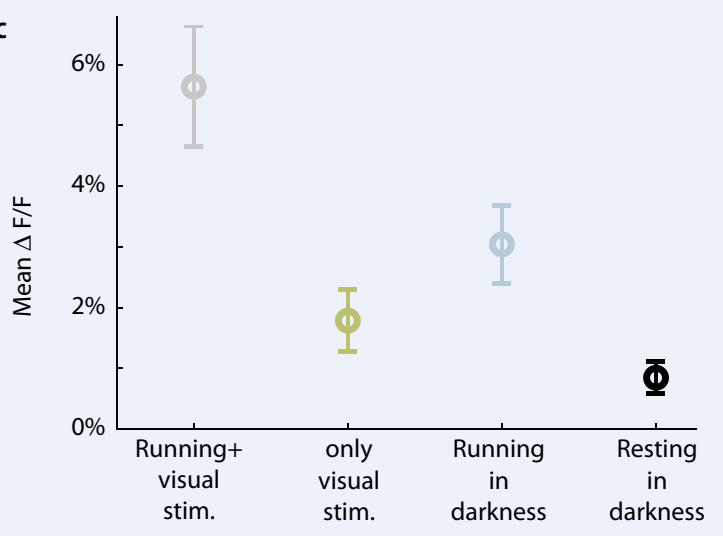

d

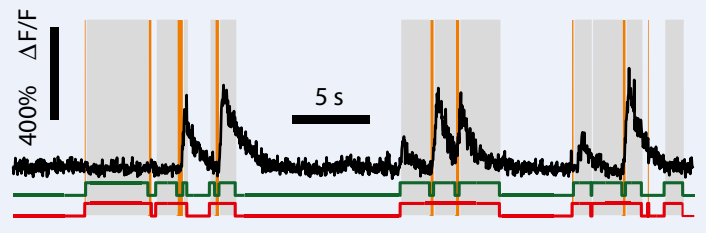

e

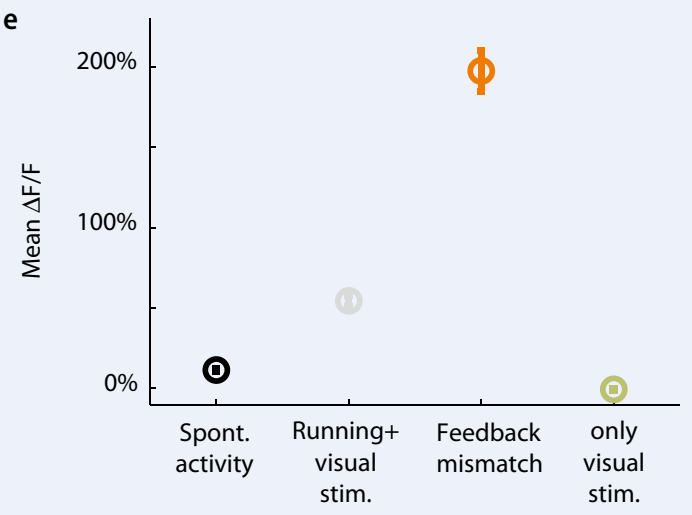

Fig. $4 \Delta$ Interaction of sensory and motor signals in the mouse visual cortex. a Schematic of experimental set-up. The mouse is head-fixed with a head-post under a two-photon microscope (not shown). An air-supported Styrofoam ball enables the animal to run while remaining stationary. The rotation speed of the ball is optically monitored and controls-in closed-loop mode - the optic flow of the visual stimulus that is presented on monitors on both sides of the mouse. A cover above the ball prevents the animal from receiving "true" visual feedback. In these experiments, rotation of the ball around the vertical axis was blocked. $\mathbf{b}$ Calcium signals of a GCaMP3-expressing layer 2/3 neuron in the visual cortex under different experimental conditions. The top trace shows fluorescence changes $(\Delta F / F)$ in closed-loop mode: The neuron is active while the animal is running and receives visual feedback (gray areas). The bottom trace shows that the same neuron also responds, albeit weaker, to passive visual stimulation, i.e., when the mouse is not running (green areas). Green trace: binary vector for visual flow; red trace: binary vector for mouse running. c Mean response amplitudes (averaged over 269 neurons) for different stimulus conditions. Visual stimulation during running induced the largest activity, while stimulation at rest evoked relatively weak signals, which on average were even lower than signals evoked during running in the dark. $\mathbf{d}$ Example neuron showing pronounced responses to brief halts of the visual flow during running (orange areas). e Mean response amplitudes of the neuron in $\mathbf{d}$ for different stimulus conditions. By far, the largest responses occurred following the brief halts of the visual flow during running, i.e., for abrupt mismatches between the expected and the real input ("feedback mismatch"). (Adapted from [3])

neurons (• Fig. 4). Apparently, these responses are driven by the mismatch between actual and expected visual feedback. Such a mismatch error signal could be used, for example, to correct motor control signals. Although many questions remain unanswered, for instance, whether the signals correlating with motor activity indeed represent an efference copy of mo- tor commands, these experiments clearly demonstrate that even in primary cortical areas sensory processing is not merely a passive process, but also reflects a comparison between actual and expected sensory signals.

Calcium imaging experiments in awake animals are also ideally suited to studying neuronal representations in the motor cortex, because simultaneous imaging of the activity of numerous neurons allows for a better understanding of population codes in the control of movements. Again using the genetically encoded indicator GCaMP3, Huber and colleagues studied the stability and plasticity of neuronal responses in the motor cortex of mice [2]. To this end, head-fixed 
animals were trained to detect an object with their whiskers and report detection by licking in order to receive a water droplet as reward. Simultaneously, calcium signals of motor cortex neurons were measured with two-photon microscopy. The authors found that the recorded population activity was well suited to predict salient behavioral parameters, such as the time of whisker-object contact or the degree of whisker curvature changes. Interestingly, even a few neurons were sufficient to reach near-optimal prediction, indicating redundancy in coding. As the same neurons were chronically recorded during training for up to 2 weeks, changes in the responsiveness of single neurons and the population with increasing learning success could also be analyzed. The results showed that some correlations between neuronal activity and specific behavioral aspects were stable, whereas other parameters systematically changed during learning. For instance, the activation of licking-related neurons shifted to progressively earlier times after detection of the object. Apart from elucidating signal processing in the motor cortex, these experiments highlight perhaps the most significant potential of genetically encoded calcium indicators, namely, the possibility to reveal changes in neuronal dynamics over long time periods during learning.

\section{Conclusions, open questions, and outlook}

The development of genetically encoded calcium indicators and their combination with high-resolution imaging methods, such as two-photon microscopy, have opened many new possibilities to study neuronal network activity, especially under behaviorally relevant conditions when the networks under study are in action. These promising new experimental opportunities provide fundamental insights into the operation of local microcircuits and could also help bridge the levels of single-cell integration and macroscopic network activity in connected brain regions. Further development of protein-based indicators is ongoing, and additional improved calcium indicators and membrane potential indica- tors will become available soon. However, when using these new indicators, one aspect to keep in mind is whether and to what extent their expression may affect the activity of neurons or local networks. Besides the possibility of toxicity, for example, after virus injection, potential alterations in intracellular calcium dynamics caused by indicator buffering could be problematic. For example, as an intracellular messenger, calcium ions are crucially involved in synaptic plasticity. Depending on the expression method and the resulting protein concentration, possible effects of indicator expression should be tested, especially the occurrence of potentially harmful phenotypes in transgenic mice. In their study, Huber et al. [2] could show that a concentration of $15 \mathrm{mM} \mathrm{GCaMP3}$ does not affect longterm potentiation in brain slices. Staying within certain indicator concentration ranges is certainly recommended. An important future goal is to target functional studies to specific cell types, by selective indicator expression in particular cells, or by marking them with additional fluorescent proteins, or by post hoc immunohistochemistry. Functional characterization of different subtypes of inhibitory interneurons, which presumably are highly relevant for local signal processing, is of utmost importance. Moreover, well-defined populations of projection neurons, which embed local circuits into larger networks of communicating brain regions, can be identified by retrograde transport of tracers, including viruses. Finally, calcium indicators are also suitable for studying the activity of glial cells, for example, astrocytes and microglia. And on a subcellular scale, it should soon become possible to carry out long-term functional imaging of presynaptic nerve terminals, postsynaptic dendritic spines, or astroglial endfeet. Not only indicators, but also imaging technologies are undergoing continued further development. One major goal is to improve the temporal resolution of fluorescence measurements, in order to obtain more precise timing information about action potential patterns. To this end, minimization or compensation of motion artifacts, especially in awake, behaving animals, will be crucial. Moreover, imaging of ever-larger neuronal populations (at many imaging depths) is desirable to obtain an as-complete-as-possible view of network dynamics. Within the next few years it seems feasible to achieve in vivo imaging of several thousand cells, albeit probably at relatively low acquisition rates. Furthermore, recently developed methods for visualizing deeper brain structures with miniature endoscopic optics will be further optimized.

Finally, it will be necessary to move beyond purely descriptive studies in order to attain a deeper understanding of neuronal circuit function. New anatomical methods promise to reveal connectivity in neuronal networks in a comprehensive manner, which should allow for correlating structural aspects of neuronal wiring with functional microcircuit properties. Furthermore, combining functional imaging with novel optogenetic methods, that is, optical excitation or inhibition of specific neurons or sub-populations with light-activatable proteins, should help to move beyond a purely correlative description and to elucidate causal relationships between network activity and behavior.

\section{Corresponding address}

Prof. Dr. F. Helmchen

Brain Research Institute,

University of Zurich

Winterthurerstrasse 190, 8057 Zurich

Schweiz

helmchen@hifo.uzh.ch

F. Helmchen. Prof. Fritjof Helmchen studied physics and medicine (theoretical part) at Heidelberg University. He carried out his diploma and dissertation work in the department of Prof. B. Sakmann at the Max Planck Institute of Medical Research in Heidelberg (PhD degree 1996 at the University of Göttingen). Following a 3-year postdoc with Prof. W. Denk at the Bell Laboratories in New Jersey, he returned to the Max Planck Institute in Heidelberg as junior group leader and completed his Habilitation in Physiology in 2004. Since 2005, he has been Professor and Co-Director of the Brain Research Institute at the University of Zurich.

Prof. Dr. M. Hübener

Max Planck Institute of Neurobiology Am Klopferspitz 18, 82152 Martinsried mark@neuro.mpg.de 
M. Hübener. Prof. Mark Hübener studied biology in Tübingen and Hamburg. He finished his PhD in 1991 in Tübingen at the Friedrich Miescher Laboratory of the Max Planck Society and subsequently joined the Max Planck Institute of Neurobiology in Martinsried as a research assistant. He completed his Habilitation in Zoology at the Ludwig Maximilian University in Munich, and since 2002 has headed a research group in the Department of Synapses-Circuits-Plasticity at the MPI of Neurobiology. Since 2006, he has been Affiliate Professor at the LMU Munich.

Conflict of interest. On behalf of all authors, the corresponding author states that there are no conflicts of interest.

\section{References}

1. Dombeck DA, Khabbaz AN, Collman F, Adelman TL, Tank DW (2007) Imaging large-scale neural activity with cellular resolution in awake, mobile mice. Neuron 56:43-57

2. Huber D, Gutnisky DA, Peron S, O'Connor DH, Wiegert JS, Tian L, Oertner TG, Looger LL, Svoboda $\mathrm{K}$ (2012) Multiple dynamic representations in the motor cortex during sensorimotor learning. Nature 484:473-478

3. Keller GB, Bonhoeffer T, Hübener M (2012) Sensorimotor mismatch signals in primary visual cortex of the behaving mouse. Neuron 74:809-815

4. Knöpfel T (2012) Genetically encoded optical indicators for the analysis of neuronal circuits. Nat Rev Neurosci 13:687-700

5. Looger LL, Griesbeck O (2012) Genetically encoded neural activity indicators. Curr Opin Neurobiol 22:18-23

6. Lütcke H, Murayama M, Hahn T, Margolis DJ, Astori S, Meyer zum Alten Borgloh S, Göbel W, Yang Y, Tang W, Kügler S, Sprengel R, Nagai T, Miyawaki A, Larkum ME, Helmchen F, Hasan MT (2010) Optical recording of neuronal activity with a genetically encoded calcium indicator in anesthetized and freely moving mice. Front Neural Circuits 4(9):1-12

7. Mank, M, Santos AF, Direnberger S, Mrsic-Flogel TD, Hofer SB, Stein V, Hendel T, Reiff DF, Levelt C, Borst A, Bonhoeffer T, Hübener M, Griesbeck O (2008) A genetically encoded calcium indicator for chronic in vivo two-photon imaging. Nat Methods 5:805-811

8. Margolis DJ, Lütcke H, Schulz K, Haiss F, Weber B, Kügler S, Hasan MT, Helmchen F (2012) Reorganization of cortical population activity imaged throughout long-term sensory deprivation. Nat Neurosci 15:1539-1546

9. Minderer M, Liu W, Sumanovski LT, Kügler S, Helmchen F, Margolis DJ (2012) Chronic imaging of cortical sensory map dynamics using a genetically encoded calcium indicator. J Physiol 590:99-107

10. Miyawaki A, Llopis J, Heim R, McCaffery JM, Adams JA, Ikura M, Tsien RY (1997) Fluorescent indicators for $\mathrm{Ca} 2+$ based on green fluorescent proteins and calmodulin. Nature 388:882-887

11. Niell CM, Stryker MP (2010) Modulation of visual responses by behavioral state in mouse visual cortex. Neuron 65:472-479

12. Tian L, Hires SA, Mao T, Huber D, Chiappe ME, Chalasani SH, Petreanu L, Akerboom J, McKinney SA, Schreiter ER, Bargmann Cl, Jayaraman V, Svoboda K, Looger LL (2009) Imaging neural activity in worms, flies and mice with improved GCaMP calcium indicators. Nat Methods 6:875-881 